\title{
Negatively-stained polysomes on rough microsome vesicles viewed by electron microscopy: further evidence regarding the orientation of attached ribosomes
}

\author{
A. Kent Christensen \\ Department of Anatomy and Cell Biology, Medical Science II Building, University of Michigan Medical School, \\ Ann Arbor, MI 48109-0616, USA \\ Received: 15 December 1992 i Accepted: 22 October 1993
}

\begin{abstract}
Rough microsomes, derived from rough endoplasmic reticulum of rat liver, were studied by electron microscopy after negative staining, to seck further information about the orientation of ribosomal small and large subunits in bound polysomes. Rough microsomal vesicles were fixed with $2 \%$ formaldehyde, centrifuged onto electron-microscopic grid membranes, and were then negatively-stained with $2 \%$ phosphotungstic acid. In these preparations, viewed with the electron mieroscope, flattened rough microsomal vesicles with bound polysomes were sometimes discernible, and the individual ribosomes in the polysomes occasionally showed small and large subunits. The small subunits were uniformly oriented toward the inside of the polysomal curve. The large and small subunits appeared to be alongside one another on the membrane, consistent with the orientation that has been described by Unwin and his co-workers. The boundary between the small and large subunits occurred at approximately the same level in the ribosome where inter-ribosomal strands have been described previously in surface views of bound polysomes in positivelystained electron-microscopic tissue sections. This further confirms the identity of the strands as messenger RNA.
\end{abstract}

Key words: Polysomes - Ribosomes - Subunits - Liver Electron microscopy - Negative stain - Rat (SpragueDawley)

\section{Introduction}

Polysomes that are bound to membranes of the rough endoplasmic reticulum (RER):produce most secretory, membrane, and lysosomal proteins. The detailed orientation of the individual ribosomes in bound polysomes has been of interest over the years. More recent findings are those of Unwin and his coworkers (Unwin and Taddei

This work has appeared in abstract form: Christensen AK (1990)
1977; Unwin 1977, 1979; Kühlbrandt and Unwin 1982; Milligan and Unwin 1986), who studied 2-dimensional crystals of ribosomes bound to RER membranes in oocytes of hibernating lizards. Electron micrographs of the crystals were analyzed by optical diffraction and Fourier synthesis to yield 3-dimensional reconstructions of the ribosomes. These showed that the large and small ribosomal subunits were arranged alongside one another on the RER membrane. This model differed markedly from the traditional view that the small subunit was situated on top of the large subunit, away from the membrane (see Results and Discussion). These studies also showed that the long axis of the small subunit was approximately parallel to the RER membrane. Tests on the binding characteristics of the crystalline ribosomes gave essentially the same results as had been obtained for normal bound ribosomes from secretory cells, suggesting that the binding of the crystalline ribosomes to the RER membrane, and of the ribosomal subunits to each other, was essentially normal.

Since the crystalline ribosomes of the Unwin studies were not involved in translation, they were not organized into polysomes. It was not possible, therefore, to relate the orientation of the ribosomes to the overall organization of bound polysomes. In the present study, an in vitro approach has been used to examine surface views of polysomes on flattened, negatively-stained rough microsome vesicles vicwed by electron microscopy (EM). The lindings indicate that the ribosomal subunits are arranged side-by-side on the RER membrane, consistent with the results of Unwin's group (described above), and that the small ribosomal subunits are oriented toward the inside of the polysomal curve.

\section{Materials and methods}

Animals

Mature Sprague-Dawlcy rals (Charles River Breeding Laborato. ries, bratnch office in West Pottage, Mich.) werc mairlained on a 
$13 \mathrm{~h}$ light, $11 \mathrm{~h}$ dark regimen, and fed water and rat chow ad libitum.

\section{Isolation of rough microsomes}

Rough microsumes (RM) were prepared from rat liver as described by Gaetani et al. (1983), using a triethanolamine (TEA) homogenization buffer (Walter and Blobei 1983), consisting of $250 \mathrm{mM}$ sucrose, $50 \mathrm{mM}$ TEA, $50 \mathrm{mM}$ potassium acetate, $6 \mathrm{mM}$ magnesium acetate, $1 \mathrm{mM}$ EDTA, $1 \mathrm{mM}$ dithiothreitol (DTT), and $0.5 \mathrm{mM}$ PMSF, with a final pH of 7.6. Briefly, $1-2 \mathrm{~g}$ of tissue was diced in $4 \mathrm{X}$ cold homogenization buffer ( $w \mathrm{t} / \mathrm{vol}$ ), and was then homogenized by about 5 passes in a Potter-Flvejhem tissue homogenizer. Partial protcction from ribonuclease digestion was provided by care with glassware and handling as well as by the endogenous ribonucleasc inhibitor present in rat liver (Gaetani et al. 1983). The homogenate was centrifuged for $10 \mathrm{~min}$ at $8000 \mathrm{RPM}$ in a Sorvall RC2-B refrigerated centrifuge, using an SS-34 rotor. The supernatant was decanted and centrifuged again for $15 \mathrm{~min}$. The resulting postmitochondrial supernatant contaithed predominantly rough and smooth microsomes derived from fragmented rough and smooth endoplasmic reticulum. Rough microsomes were then isolated (Gaetani et a1. 1983) on a 3-layer discontinuous sucrose gradient $(1.35,1.55,1.8 \mathrm{M}$ sucrose) by centrifugation with a Beckman L8-70 ultracentrifuge (SW-40TI rotor) for $12 \mathrm{~h}$ at $36000 \mathrm{RPM}$ and $4^{\circ} \mathrm{C}$. The "heavy" rough microsomal fraction was mixed $1: 1$ with cold glycerol (Ajdrich, Milwaukec, Wis., specttophotonet ic grade, No. 19 161.2), and $200 \mu 1$ aliquots were stored in cryogenic tubes in a liquid nitrogen refrigerator $\left(-196^{\circ} \mathrm{C}\right)$. This storagc allowed subsequent processing to be carried out more canveniently. In previous biochcmical work (Kreibich et al. 1978, 1983; Gaetani et al. 1983; Walter and Blobel 1983), RM and stripped RM have been stored routinely in cryoprotectant ( $50-66 \%$ glycerol or $250 \mathrm{mM}$ sucrose) at $-70^{\circ} \mathrm{C}$ or $-80^{\circ} \mathrm{C}$, with little or no loss of activity for later in vitro protein translation. Glycerol (up to $40^{\circ} \%$ ) is sometimes used to stabilize native protein structure (Gekko and Timasheff 1981 ), and has been utilized for this purpose in biochemical studics involving RM (Yu et a.. 1989).

\section{Application to EM grid membranes, negative staining. and electron microscopy}

An aliquot of RM stuck was brought to $-20^{\circ} \mathrm{C}$, and a $5 \mu$ sample was taken up with a Pipetman and added to $1 \mathrm{ml}$ of cold TEA buffer (same as the bomogenizing buffer described above, but lacking EDTA, DTT, and PMSF), with brief manual mixing. The matcrial was then fixed in $2 \%$ paraformaldehyde by mixing cqual parts of the above solution with cold $4 \%$ paraformaldehyde (in the same TEA buffer), mixing manually. After $0.5-1 \mathrm{~h}$ of fixation at $4^{\circ} \mathrm{C}$, $100 \mu$ of the solution was added to each of several cold "platform microtubes", which were $1.8 \mathrm{ml}$ microtubes containing a plug of polymerized epoxy resin (Spurr 1969) at their tips. $\Lambda \mathrm{n}$ EM grid was inserted into the solution in each tube and was left face-up on the surface of the plug. The grids had been coated previously with a Formvar membrane and carbon, and were glow-discharged just before usc to make the membrane sutface more hydrophilic. The tubes were centrifuged for $10 \mathrm{~min}$ at $4^{\circ} \mathrm{C}$ in a Microfuge $B$ (Beckman), at about $8700 \times g$, to aid in bringing $\mathrm{RM}$ vesicles down on the EM grid membrane.

The grids were then washed $1 \mathrm{~min}$ in double-distilled watcr at room temperature. Since subsequent steps would involve floating the grids face down on drops of fluid, it was necessary at this point to dry the back of each grid, without drying the face. This was accomplished by placing a grid face down in a $10 \mu \mathrm{l}$ droplet of distilled water on paraflm, and genily applying a piece of Whatman No. 2 filter paper to the grid, absorbing the water from the back of the grid, but leaving fluid between the grid and the parafilm. The grid was then grasped with jeweler's forceps and quickly floated face down on another drop of distilled water, remaining there for 1 min. For negative staining (Shelton and Kuff 1966), the grids were floated for $2 \times 1 \mathrm{~min}$ at room temperature on drops of $2 \%$ phospliotungstic acid (in double-distilled water. $\mathrm{pH} 5.8$, containing $5 \mathrm{mM}$ $\mathrm{MgCl}_{2}$ ). Finally, each grid was picked up with jeweler's forceps and the edge was brought obliquely against a piece of filter paper for a moment, to draw off excess staining solution, after which the grid was air-dried. Grids were viewed with a Philjps 201 clectron-microscope operated at $60 \mathrm{kV}$, and most micrographs were taken at $20000 \times$ initial magnification (checked with a catbon grating replica).

\section{Conventional EM of rat pituitary}

Pituilary tissue from rats was fixed, embedded, sectioned, and positively stained for electron microscopy by conventionat mothods, as described previously (Christensen et al 1987).

\section{Results and discussion}

In these RM preparations, viewed with the electron microscope, many of the vesicles were flattened, and polysomes were often discernible.

Fig. $1 \mathrm{a}$ and $\mathrm{b}$ show negatively-stained polysomes on flattened RM vesicles, from material prepared as described above. The individual ribosomes, which were white because of the negative staining, generally consisted of 2 subunits, one larger than the other. Labeled drawings above the 2 figures may aid in their interpretation. In most of the ribosomes, the smaller subunit was oriented toward the inside of the polysomal curve.

As a frame of reference, these negatively-stained images of bound polysomes prepared in vitro may be compared with the well-known positively-stained polysome surface views that are seen occasionally in conventional EM tissue sections when the RER happens to be cut in grazing section (Palade 1955). Fig. $1 \mathrm{c}$ and $\mathrm{d}$ show examples of positively-stained polysome surface views from tissue sections of rat pituitary, similar to those described in our previous study (Christensen et al. 1987). Labeled drawings below the positively-stained figures may aid in their interpretation. In prior detailed descriptions of positively-stained polysome surface views (see particularly Bonnett and Newcomb 1965; David and Metzler 1967; Christensen et al. 1987), the individual ribosomes usually appeared oblong, with the long axis oriented perpendicular to the presumed path of the mRNA. The end of the ribosome that was oriented toward the inside of the polysomal curve was usually wider than the outer ond. These features can be observed in Fig. Ic and d.

Ribosomal subunits were not visible in the positivelystained surface views of the present study (Fig. $1 \mathrm{c}$ and d), consistent with most past literature. In general, polysomal appearance and the spacing of the ribosomes were similar in the negatively-stained and positively-stained polysomes, which are shown here at the same magnification. However, the negatively-stained ribosomes (Fig. 1 a and b) appeared somewhat smaller than those that were positively stained (Fig. $1 \mathrm{c}$ and d). This is to be expected in negative staining, since the stain may overlap boundaries, 

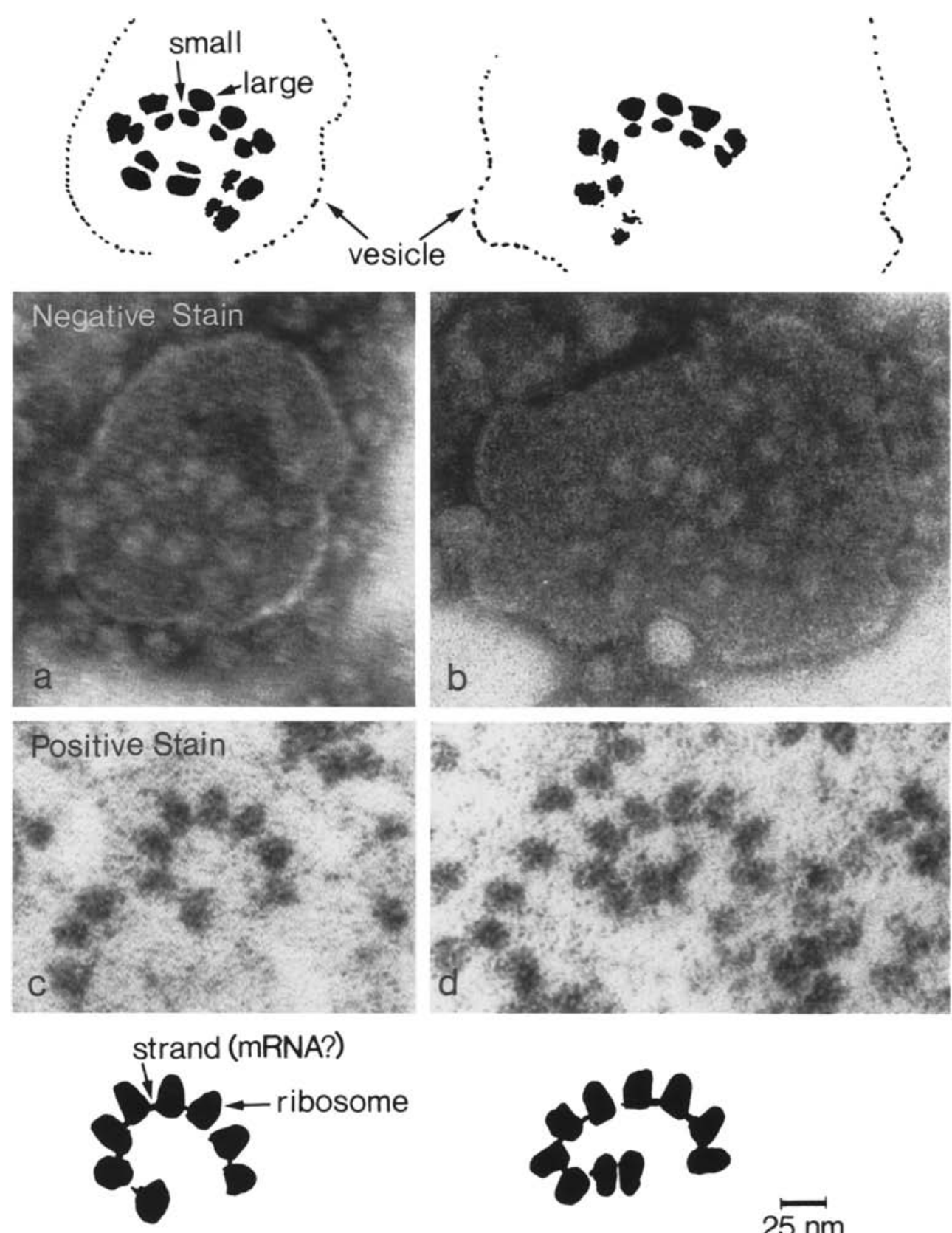

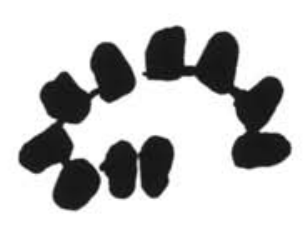

$2 \longdiv { 5 \mathrm { nm } }$
Fig. 1a, b. Negatively-stained bound polysomes on flattened rough microsome vesicles from rat liver. Diagrams above figures show pertinent structures and labels. Vote large and small ribosomal subunits (appearing white as a result of negative staining). The small subunit is consistently oriented toward the inside of the polysomal curve. $\times 230000$. c, d Examples of positively-stained bound polysomes in conventional tissue scctions of a somatotrope from rat pituitary, provided to allow comparison with above negative-stain results (see text). Diagrams below figures show pertinent structures and labels. Note appearance of ribosomes and the strand that somelimes passes between them. Ribosomal subunits cannot be distinguished in these positively-stained preparations. $\times 230000$ thus making the ribosome and its subunits appear smaller.

In the literature on surface views of positively-stained polysomes in tissue sections, a thin strand has occasionally been described extending between ribosomes (Watson 1959; Ross and Benditt 1964; David and Metzler 1967; Bielka 1982 [his Fig. 4 b, EM by David]; Christensen et al. 1987). The strand usually was seen to contact the ribosomes near their innor ends. Examples of this strand are shown in Fig. $1 \mathrm{c}$ and d. It has seemed reasonable in the past to suggest that the strand represented the mRNA, perhaps thickened by secondary RNA structure or adsorbed protein, but no particular evidence for this interpretation has been available previously. The strands were not visible in the negatively-stained polysomes of the present study (Fig. 1 a and b). However, the interlace between small and large subunits occurred at approximately the same position on the negatively-stained ribosome where the strands were found in the positivelystained preparation. This would support the view that the strands represent mRNA, which is known to pass between the small and large ribosomal subunits.

The negative-stain images of the present study seem consistent with the side-by-side model for subunit arrangement in bound ribosomes, but not with the traditional model. In negative staining, the stain accumulates around structures of interest, which are then seen in negative image. Fig. 2 shows how the stain would presumably be distributed on a ribosome oriented according to the traditional model (upper left) or to the side-by-side model (upper right). Below each diagram is the appearance that would be expected if the negatively-stained ri- 
Traditional Model

\section{Probable \\ negative-stain distribution distribution}
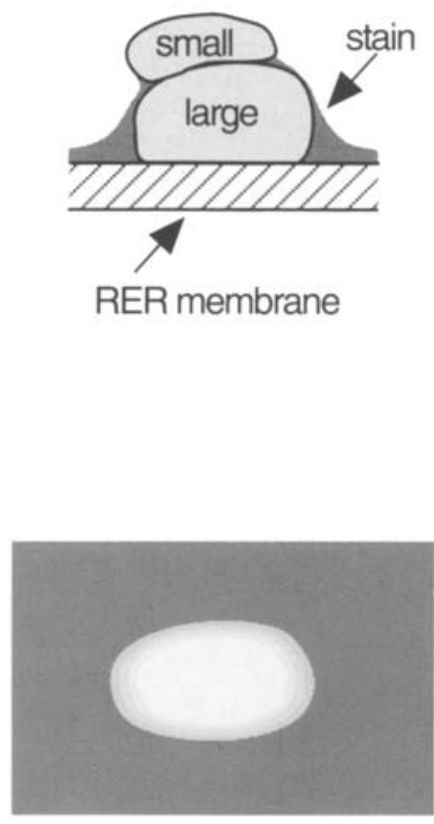

Side-by-side Model
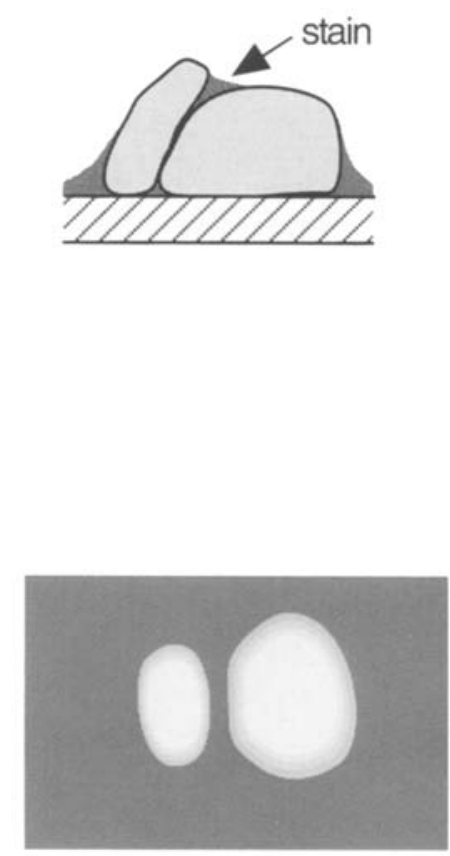

Fig. 2. Diagrams illustrating 2 main models for ribosomal orientation on the membrane of rough $\mathrm{ER}$, and how ribosomes might be expected to appear in negativelystained preparations. In the traditional model (upper lefi), the large subunit is in contact with the RER membrane and the smail subunit lies on top of the large subunit, away from the membrane. Negative stain ldark shading) would accunnulate at the sides of the ribosome. In the side-byside nodel (upper right), the 2 subunils occut alongside one another on the membrane, and negative stain may be visible between subunits. Below each diagram is a representation of how a negatively-stained ribosome arranged according to that model might be expected to appear in EM, when viewed from dircetly above, the vantage point in the present study. Negatively-stained ribosomes in Figures $1 a$ and $1 b$ appear as would be expected for the side-by-side model bosome were viewed from directly above with the electron-microscope, as in the present study. In the traditional model, the large subunit is attached to the RER membrane and the small subunit is situated on top of the large subunit, away from the membrane. The negative stain would be gathered primarily around the sides of the ribosome. When viewed from above, one would expect the negative stain image to appear as a single oval. On the other hand, in the side-by-side model, the subunits are arranged alongside one another on the RER membrane, and stain may penetrate between the subunits. When viewed from above, the stain lying between the subunits would cause the small and large subunits to be seen individually. The negatively-stained ribosomes in Fig. 1 a and $b$ have the appearance that would be expected for the side-by-side model.

The side-by-side model (Unwin and Taddei 1977; Unwin 1977) arose from studies on 2-dimensional ribosomal crystals, in which the ribosomes were not involved in translation. The present findings offer evidence that the model is also valid for organized bound polysomes. When the 3-dimensional ribosomal reconstruction of Milligan and Unwin (1986), produced at $55 \AA$ resolution, is viewed from above (their Fig. 3 b), it resembles the average appearance of positively-stained ribosomes in the surface views of polysomes from tissue sections (our Fig. 1c and d). The boundary between large and small subunits (arrowheads in their Fig. 3 a) is consistent with the boundary position in our negatively-stained ribosomes (our Fig. $1 \mathrm{a}$ and $\mathrm{b}$ ) and with the strand position in the positively-stained polysomes (our Fig. $1 \mathrm{c}$ and d). Verschoor and Frank (1990) have produced a more detailed 3-dimensional reconstruction of free (unbound) $80 \mathrm{~S}$ ribosomes at $37 \AA$ resolution. However, the orientation that those free ribosomes would have if they were bound to the RER membrane has not been established. Those authors showed considerable space between the large and small subunits, except near the base, where there is tight contact. The substantial negative stain between subunits in our micrographs would be consistent with that feature.

Our results do not appear to be consistent with the traditional model for the orientation of bound ribosomes. The development of the traditional model was based primarily on biochemical studies of subunit binding to the RER membrane, and on electron micrographs of ribosomes seen in side view. Sabatini et al. (1966) showed biochemically that the large ribosomal subunits were firmly attached to the RER membrane, while the small subunits were only weakly attached to the membranc, if at all. When rough microsome (RM) vesicles were negatively stained on EM grid membranes, and then viewed with the electron microscope (Sabatini et al. 1966; Shelton and Kuff 1966), no interpretable images of ribosomal subunits or of organized polysomes were distinguished on the exposed face of RM vesicles. However, ribosomes were observed in side view; attached to the edges of the flattened vesicles. These ribosomes had a bipartite appearance, and the larger subunit was attached to the edge of the vesicle, while the smaller subunit was on top of the larger one, away from the vesicle edge. The boundary between large and small subunits was approximately parallel with the edge of the vesicle. Although conventional electron micrographs of positivelystained ribosomes in tissue sections rarely showed distinct ribosomal subunits, occasional micrographs were published in which apparent large and small subunits could be distinguished in ribosomes bound to the RER, 
when the RER membrane was observed edge-on, and the ribosomes were therefore seen in side view (Palade 1967, 1975; Florendo 1969; David and Uerlings 1970). In those instances, again, the large subunit was situated on the RER membrane, and the small subunit formed a roof over the large subunit and was not in contact with the membrane. However, the paper by David and Uerlings (1970) also included occasional ribosomal side-views in which both subunits appeared to be in contact with the membrane.

In the literature, positively-stained polysomes seen in surface views of the RER in tissue sections (similar to those shown here in Fig. $1 \mathrm{e}$ and d) have almost never exhibited distinguishable ribosomal subunits. However, there are 2 exceptions. The paper by Florendo (1969) included a surface vicw of a partial polysome (his Fig. 2), with subunits visible in 3 ribosomes. The smaller subunits were oricnted toward the inside of the polysomal curve. He commented that the micrograph suggested "that both large and small subunits are closely apposed to the underlying membrane." I Iowever, the appearance of these ribosomes, seen from above, was the same as that of ribosomes shown in side view elsewhere in the paper, which was not what would have been anticipated. To reconcile this apparent inconsistency, he postulated that the ribosomes were actually oriented obliquely, and that the "small subunit-large subunit axes of ribosomes arranged in whorls [polysomes] are disposed in three dimensions on the surface of a truncated cone." The surface view in his paper did not happen to show a strand. The other paper, by Bonnett and Newcomb (1965, their Fig. 10, inset), also included ribosomal subunits in surface views of bound polysomes. However, the subunits appeared to be about the same size, and so it was difficult to draw conclusions about the orientation of the small subunits. A freeze-fracture study on rough microsome vesicles, secn in surface view after deep etching (Ojakian et al. 1977, their Tig. 3), showed grooves across 2 of the bound ribosomes, defining subunits. Again, the subunits were of approximately equal size.

It may be of interest to note that the appearance of ribosomes in our negatively-stained preparations of bound polysomes is similar to results ubtained by Shelton and Kuff (1966), in which small, free (uvbound) polysomes were brought down on EM grid membranes and negatively stained. The intrinsic geometry of these free polysomes caused them consistently to assume a round or C-shaped configuration on the EM grid membrane, and the small ribosomal subunits were uniformly visible and were oriented toward the inside of the polysomal curve. The bound polysomes of the present study have a similar appearance on the membranes of flattened RM vesicles. This implies that the shape of small, bound polysomes, and the orientation of their ribosomes, may reflect inherent characteristics of polysomal organization.

Some possible concerns need to be considered in the interpretation of the negalively-stained images in the present study. One concern is that the side-by-side arrangement of subunits might have been produced as a drying artifact during negative staining, if a small subunit that was originally on top of the large subunit would be drawn down to the membrane by surface tension or other forces during drying. Air-drying during negative staining lends to flatten structures to some degree, although the distortion is not nearly as severe as that which occurs during simple air drying, since the negative stain gathers around small structures, forming a coat that partially protects them from the damaging effects of aqueous surface tension. Lake (1978) used tilting experiments to compare the extent of tlattening in 3 different negative-staining approaches (double carbon, single carbon, droplet), and found that the longest dimension of the bacterial ribosomes varied from $180 \AA$ to $250 \AA$ in the 3 methods, indicating a considerable difference in flattening. However, the flattening did not seem to have an appreciable effect on structural detail seen in the negatively-stained ribosomes, since similar features were observed by all 3 methods. According to Hayat and Miller (1990), fiattening of negatively-stained structures usually makes them appear somew hat larger than the corresponding fresh or positively-stained structures. Comparing the negativelystained ribosomes in Fig. $1 \mathrm{a}$ and $\mathrm{b}$ with the positivelystained ribosomes in Fig. $1 \mathrm{c}$ and d shows that this is not the case in the present material, suggesting that flattening was not excessive.

If the binding between small and large subunits were unstable, then one could imagine an artifactual shift in the position of the small subunit during negative staining. However, this binding appears generally to be quite stable during negative staining, judging by the small number of detached subunits seen in fields of negatively-stained free ribosomes (monosomes) in bacteria (Lake 1981) and in eukaryotes (Verschoor and Frank 1990). If the binding between subunits in monosomes is relatively stable, then subunit binding should be even more stable in ribosomes that are organized into bound polysomes, where the small subunit would be further stabilized by the anchoring effect of the mRN $\wedge$ and of the nascent polypeptide. The small subunit binds the mRNA and tRNAs, and it might be expected, therefore, that formaldehyde fixation of a bound polysome would stabilize the mRNA and polypeptidyl-tRNA in the ribosomes. This should limit considerably the mobility of the small subunit, and thus make it even less likely to shift its position relative to the large subunit during negative staining.

In view of the above considerations, it seems unlikely in the present study that the side-by-side appearance of subunits in the ribosomes of Fig. $1 \mathrm{a}$ and $\mathrm{b}$ would have resulted from detachment of small subunits from the top of large subunits during negative staining, and their movement down to the membrane, all on the same finward) side of the ribosomes. It would seem more likely that the subunits were side-by-side before negative staining was carried out.

Another possible concern is that the side-by-side appearance might be a tilting artifact. If a ribosome whose small subunit was actually on top of the large subunit happened to be viewed from a tilted perspective, then the subunits might appear, mistakenly, to be side-by-side on the membrane. This is unlikely in the present study, since the ribosomes are all oriented with the same side up on 
the $R M$ vesicle (RER) membranc, and are being viewed from directly above, without any tilt. The ribosomes should have a consistent orientation on the RM vesicle membrane because they are attached to it by their specific attachment sites (Unwin 1979; Savitz and Meyer 1993). The $R M$ vesicle membrane is being viewed from directly above because the vesicles have been flattened down on the EM grid membrane, which is in a plane perpendicular to the viewing axis. It does not seem likely, therefore, that the appearance of these ribosomal images could be ascribed to a tilt.

Acknowledgenents. The able technical assistance of Jami Grossfield and Kyung-mi Lim in this study is greatly appreciated. I want to thank Catol M. Bourne for her expert help with pituitary polysomes. I am also grateful to Elaine M. A. Christensen for preparing Fig. 2. Part of this study was supported by an NIH Biomedical Research Support Grant, RR 05383.

\section{References}

Bielka $\mathrm{H}$ (ed) (1982) The eukaryotic ribosome. Springer, Berlin

Bonnett HT Jr, Newcomb EH (1965) Polyribosomes and cisternal accumulations in root cells of radish. J Cell Biol 27:423-432

Christensen AK (1990) The small subunits of ribosomes are oriented toward the center of bound polysomes on the rough endoplasmic reticulum (abstract). $\Lambda$ nat Rec $226: 20 \mathrm{~A}$

Christensen AK, Kahn LE, Bourne CM (1987) Circular polysomes predominate on the rough endoplasmic reticulum of somatotropes and mammotropes in the rat anterior pituitary. Am I Anal 178:1-10

David H, Metzicr E (1967) Anordnung und Aufbau der Polysomen in der intakten Rattenleberzelle. Acta Biol Med Germ 18:529 540

David $H$. Uetlings I (1970) Ribosomen-Membran-Beziehungen und ribosomale Substrukturen in intakten Rattenleberzellen. Acta Biol Med Germ 25:343-350

Florendo NT (1969) Ribosome substructure in intact mouse liver cells. J Cell Biol 41:335-339

Gaetani S, Smith JA, Feldman RA. Morimoto T (1983) Preparation of rough microsomes and membrane-bound polysomes that arc active in protein synthesis in vitro. Methods Enzymol 96:3-24

Gekko K, Timasheff SN (1981) Thermodynamic and kinetic examination of protein stabilization by glycerol. Biochemistry $20: 4677-4686$

Hayat MA, Miller SE (1990) Negative staining. McGraw-Hill, New York, NY

Kreibich G, Ulrich B, Sabalini D (1978) Proteins of rough microsomal membranes related to ribosome binding. I. Itentification of ribophorins I and II, membrane protein characteristics of rough microsomes. J Cell Biol 77:464-487
Kreibich G, Marcantonio EE, Sabatini DD (1983) Ribophorins I and II: membrane proteins characteristic of the rough endoplasmic reticulum. Methods Enzymol 96:520-530

Kühlbrandt W, Unwin PNT (1982) Distribution of RNA and protein in crystalline eukaryotic ribosomes. J Mol Biol 156:431448

Lake JA (1978) Electron microscopy of specific proteins: three-dimensional mapping of tibosomal proteitss using antibody labels. In: Koehler JK (ed) Advanced techniques in biological electron microscopy. II. Specific ultrastructural probes. Springer, Berlin, $173-211$

Lake JA (1981) The ribosome. Sci Am 245(2):84-97

Miligan RA, Unwin PNT (1980) Location of exit chantel for nascent protein in 80 s ribosome. Nature 319:693-695

Ojakian GK, Keibich G, Sabatini DD (1977) Mobility of ribosomes bound to microsomal membranes. A freexe-ctch and thinsection electron microscope study of the structure and fluidity of the rough endoplasmic reticulum. I Cell Biol 72:530-551

Paiade GE (1955) A small particulate component of the cytoplasm. J Biophys Biochem Cytol 1:59-68

Palade GE (1967) Cell biology, past and present. Rockefeller University Review 5:11-17

Palade GE (1975) Intracelluiar aspects of the process of protein synthesis. Science 189:347-358

Ross R, Benditt EP (1964) Wound healing and collagen formation. IV. Distortion of ribosomal patterns of fibroblasts in scurvy. I Cell Biol 22:365-389

Sabatini DD, Tashiro Y, Palade GE (1966) On attachment of ribosomes to microsomal membranes. J Mol Biol 19:503-524

Savitz AJ, Meyer DI (1993) 180-kD ribosome receptor is essential for bolh ribosome binding and protein translocation. J Cell Biol 120:853-863

Shelton $E_{3}$ Kuff EL (1966) Substructure and configuration of ribosomes isolated from mammatian cells. J Mol Biol 22:23-31

Spurr AR (1969) A low-viscosity epoxy resin embedding medium for electron microscopy. J Ultrastruct $26: 31-43$

Unwin PNT (1977) Three-dimensional model of membrane-bound ribosomes obtained by electron microscopy. Nature 269:118122

Unwin PNTT (1979) Attachment of ribosome crystals to intracellular membranes. I Mol Biol 132:69-84

Unwin PWT, Taddei C (1977) Packing of ribosomes in crystals from the lizard Lacerta sicula, J Mol Biol 114:491-506

Verschoor A, Frank J (1990) Three-dimensional structure of the mammalian cytoplasmic ribosome. J Mol Biol 214:737-749

Walter P, Blobel G (1983) Preparation of microsomal membranes for cotranslational protein translocation. Methods Exzymol 96:84-93

Watson ML (1959) Further observations on the nuclear envelope of the animal cell. I Biophysic Biochem Cylol 6:147-156

Yu Y, Zhang Y, Sabatini DD, Kreibich G (1989) Reconstitution of translocation-competent membrane vesicles from detergent-solubilized dog pancreas rough microsomes. Proc Natl Acad Sci USA 86:9931-9935e 